\title{
The selective adaptation effects of burst-cued stops
}

\author{
WILLIAM F. GANONG III \\ Massachusetts Institute of Technology, Cambridge, Massachusetts 02139
}

\begin{abstract}
One of the most compelling arguments that selective adaptation affects a phonetic level of processing is the demonstration that adaptation with burst-cued stimuli (which have no formant transitions) affects the perception of transition-cued stimuli (which have formant transitions but no bursts). Experiment 1 showed that adaptation with burst-cued [pi] and [ti] affects the perception of a (transition-cued) [mi-ni] test series, as well as a [bi-di] test series. Inasmuch as nasals never contain bursts, this demonstrates that the adaptation effect of burst-cued stops is not limited to those stimuli which normally contain bursts. If adaptation with burst-cued stops affects the perception of transition-cued stops at a more central, phonetic level of processing, their adaptation effect should transfer interaurally completely. Experiment 2 showed that the adaptation effect of both burst and transition-cued stops transfers interaurally only about one-third. These results suggest that the adaptation effect of burstcued stops is mediated by the fatigue of peripheral auditory detectors which are sensitive to both bursts and formant transitions.
\end{abstract}

The selective adaptation technique has been used to identify and investigate channels of analysis in the visual and other modalities for some time. It has been suggested that the depression in acuity caused by adaptation in these experiments is the result of the fatigue of feature detectors which mediate perception. Thus, when the selective adaptation technique was first applied to the problems of speech perception (by Eimas \& Corbit, 1973), it was natural to speculate that adaptation effects were mediated by the fatigue of feature detectors for linguistic distinctive features.

Just as simple geometric figures can be described by a few abstract features, speech sounds can be classified by relatively few linguisitic properties called distinctive features. For example, the English stops $[b, d, p, t]$ are distinguished by two distinctive features: voicing $([\mathrm{b}]$ and $[\mathrm{d}]$ are voiced, $[\mathrm{p}]$ and $[\mathrm{t}]$ are voiceless), and the place of articulation ([b] and [p] are labial, [d] and [t] are alveolar). Linguistic distinctive features are abstract descriptions of a speech sound which control its participation in phonological processes. For example, the way in

The author is now at Department of Psychology, Brown University, Providence, Rhode Island 02912. This work was part of a doctoral dissertation at the Massachusetts Institute of Technology. I would like to thank my thesis committee: Susan Carey, Joanne Miller, Merrill Garrett, and Dennis Klatt for their advice and encouragement. I would also like to thank Peter D. Eimas at Brown University for help in preparing this manuscript. This research was supported by a National Science Foundation Graduate Fellowship and a National Institutes of Health Graduate Training Program in Psychology Traineeship, NIH 5 T01 GM010 64-15. which regular nouns ending in stop consonants are pluralized depends on the voicing of the stop consonants: [s] is added to nouns ending in voiceless stops (e.g., "caps" is pronounced [k ae p s]), but [z] is added to nouns ending in voiced stops (e.g., "cabs" is pronounced [ $\mathrm{k}$ ae $\mathrm{b} \mathrm{z}]$ ). Distinctive features do not have simple acoustic characterizations. For example, many different acoustic cues have been shown to control the voicing of a particular segment (Parker, 1977; Lisker, 1975).

In "Selective Adaptation of Linguistic Feature Detectors," Eimas and Corbit (1973) showed that listening repeatedly to one sound affected the perception of that sound and of other sounds with similar feature descriptions. Thus, adaptation with [ba], for example, affected the perception of not only a [ba-pa] test continuum (by increasing the number of voiceless responses) but also a [da-ta] continuum (also by increasing the number of voiceless responses). One explanation for this result is that adaptation had fatigued the detectors for the particular values of the distinctive feature voicing. Such detectors would be sensitive to aspects of the abstract phonological description of a speech sound. However, another explanation is possible. Perhaps selective adaptation influences the auditory analysis of acoustic information which underlies the voicing decision. Both the [ba-pa] and [da-ta] test series were constructed by varying the voice-onset time (VOT) of the presented stimuli. VOT is a normal correlate of voicing in speech production, and hence a natural way to make a test continuum from [ba] to [pa] (or from [da] to [ta]) is to vary this acoustic parameter. Adapatation might then operate at an auditory level that is 
sensitive to this acoustic parameter, rather than to the phonetic voicing decision.

The question of whether adaptation influences auditory or phonetic processing stages is of interest because of the assumption (Barlow, 1972) that selective adaptation effects are mediated by the fatigue of single units. If that assumption is true, demonstrating a selective adaptation effect with a particular stimulus selectivity implies the existence of a feature detector with that stimulus selectivity. This assumption has been questioned, but no experimental evidence against it has yet been demonstrated. Thus, demonstrating that adaptation influences speech perception at a stage of processing sensitive to phonetic descriptions would strongly suggest that phonetic processing is carried out in a particularly simple way: by feature detectors for aspects of phonetic descriptions.

However, most recent work has suggested that adaptation affects primarily an auditory stage of processing. Some results have been obtained which can only be explained in terms of the similarity of the acoustic characteristics of the test stimuli used (Bailey, 1976; Ganong, 1976), and most current results can be explained in this way. But these experiments leave open the possibility that, although adaptation affects perception primarily at an auditory level, it might also affect phonetic stages of processing to a lesser degree. One recent result, the demonstration that burst-cued stops can affect the perception of transition-cued stops (Diehl, 1975; partially replicated by Ainsworth, Note 1 and Ganong, Note 2), does seem to demonstrate that adaptation also influences phonetic ${ }^{1}$ processing. The purpose of this paper is to examine this result more carefully, and to see if it can be explained without assuming the existence of phonetic feature detectors.

In natural (as opposed to synthetic) speech, many different acoustic cues are correlated with any phonetic distinction. The role of the phonetic level in speech perception is to integrate the information about the different acoustic cues in order to make a phonetic categorization of the stimulus presented. Thus, an obvious test of whether adaptation affects a phonetic level would be to find two different acoustic cues which convey the same phonetic distinction, and then determine whether adaptation with a stimulus defined by one cue affects the perception of a test continuum based on the other cue. If it does, then adaptation must have affected a level of processing which treats the two cues in the same wayand the phonetic level would be an obvious candidate.

Diehl (1975) did just this. Bursts and formant transitions are different acoustic cues which convey the place of articulation feature for stops (Hoffman, 1958). The saliency of the burst depends on its particular phonetic context, but it carries real perceptual weight in most contexts (Dorman, Studdert-Kennedy, \& Raphael, 1977). Diehl synthesized "burst-cued" [pe] and [te], which differed only in the spectra of their bursts, and a "transition-cued" [be-de] test series in which stimuli differed only in formant transitions. The sounds [p] and [b] have the same (labial) place of articulation, whereas the sounds [d] and [t] share a different (alveolar) place of articulation. Thus, Diehl predicted that, if adaptation affects a phonetic detector for place of articulation, the effect of adaptation with burst-cued [pe] should be similar (in both magnitude and direction) to that of transitioncued [be], and the adaptation effect of burst-cued [te] should be similar to that of the [de].

He found that the boundary shifts induced by adaptation with the burst-cued [te] were in the same direction (but smaller) than those obtained with the transition-cued stimuli. As a consequence, Diehl concluded that he had demonstrated that at least part of the adaptation effect operates at a phonetic level of processing.

Diehl's argument depends on two assumptions. The first is that bursts and formant transitions are different acoustic cues (i.e., information about bursts and transitions is not integrated at an acoustic level of processing). The second is that demonstrating transfer of adaptation between different cues demonstrates a phonetic adaptation effect. That is, if adaptation with a stimulus defined by one acoustic cue affects the perception of stimuli defined by a different acoustic cue, then adaptation must have affected a phonetic level of processing. ${ }^{2}$

The purpose of this study is to test these two assumptions. In order to do that, three models for Diehl's results will be described. The first, the phonetic model, is based on Diehl's assumptions. The second, the auditory model, assumes instead that burst and transitions are detected by the same auditory mechanism, and thus denies the first assumption. The third model, the prototype model, is based on denying Diehl's second assumption, and proposes a mechanism for the transfer of adaptation between different acoustic cues which does not involve the fatigue of a phonetic processing level.

The phonetic model for Diehl's results is consistent with both assumptions. In this model, there are separate acoustic detectors for bursts and formant transitions, and there is a higher level phonetic place of articulation detector which integrates the output of these detectors, and which is susceptible to adaptation. Adaptation with burst-cued stimuli affects the perception of transition-cued stimuli by changing the state of this place of articulation detector. To account for those studies which demonstrate that adaptation can influence an auditory level of processing (Bailey, 1976), this model must assume that there are auditory detectors which are also susceptible to 
adaptation. Thus, in this model, adaptation can take place at two levels, one auditory and one phonetic.

An auditory model of the transfer of adaptation between burst and transition-cued stops is even simpler than the phonetic model. It assumes that there are auditory detectors which are sensitive to both bursts and formant transitions and which are fatigued by adaptation. Thus, it denies the assumption that bursts and formant transitions are analyzed by different auditory mechanisms, that is, are effectively different acoustic cues. Two different proposals have been made for auditory mechanisms which might integrate information about bursts and formant transitions. Ades (1976) proposed that bursts and formant transitions are detected by the same lowlevel, frequency-specific auditory mechanism. Another view holds that bursts and formant transitions are both analyzed by auditory detectors which integrate information from across the whole acoustic spectrum (Blumstein, Stevens, \& Nigro, 1977).

The prototype model is based on the denial of the assumption that the transfer of adaptation between different acoustic cues must be phonetically mediated. That assumption is plausible, but not necessarily correct. It is possible, for instance, that adaptation affects the phonetic level only indirectly, through changes in its inputs. For instance, if the phonetic mechanism which analyzes place of articulation for stops sums the output of both the burst and transition detectors, the response of the phonetic level to transition-cued stimuli might be changed by changes in the tonic output of the burst detector induced by adaptation with burst-cued stops.

This counterexplanation is analogous to arguments which have been made in visual psychophysics. A seemingly natural test of whether the anatomical locus of a visual aftereffect is peripheral or central is to test whether or not the aftereffect transfers interocularly. If stimulation of one eye induces the aftereffect when that eye is closed and the test pattern is viewed through the other eye, it is natural to assume the effect is central; if not, one assumes the effect is peripheral. However, Day (1958), Delabarre (1888), and others have pointed out that this assumption is not correct. Colored afterimages (which are due to the fatigue of visual receptors in the retina) transfer interocularly. Apparently, the central mechanism which integrates the information from the two eyes is influenced by the change in the tonic output of the stimulated eye produced by prolonged viewing of a chromatic pattern, even if that eye is closed. Thus, the assumption that "transfer of adaptation between eyes is mediated by central fatigue" is not necessarily correct. The transfer may, instead, be mediated by a central integration of the output of peripheral processes which are sensitive to fatigue.
This possibility calls into question Diehl's second assumption-that the transfer of adaptation effects between different acoustic cues is mediated by fatigue at a phonetic level of processing. The prototype model explains these adaptation effects in a manner analogous to the explanation of the interocular transfer of colored afterimages given above. It assumes there are two levels of processing in speech perception. The lower level consists of detectors for different acoustic cues. The output of each detector is a graded signal indicating the likelihood that the detector's cue is a part of the stimulus presented. Adaptation is hypothesized to depress the output of those detectors which respond to the adapting stimulus. These outputs are combined by a second, integrative level of processing, which determines which stimulus was presented by comparing the output of each low-level detector with its response to prototypical members of each phonetic category. Thus, the integrative level is sensitive to just those acoustic cues which are reliable auditory correlates of each phonetic category. This integrative level is not susceptible to fatigue, but its output can be changed by adaptation-induced changes in its input. If the detector for a particular cue has been fatigued, the integrative level will receive weaker input from that detector, and therefore produce a different output.

The prototype model accounts for Diehl's results by assuming that there are separate bursts and transition detectors, and that the output of the burst and transition detectors is combined by the integrative level. Further, the prototype model also assumes that adaptation with burst-cued stops biases the tonic output of the burst detector. When transition-cued stimuli (without bursts) are presented, the tonic output of the burst detector is added to the response of the transition detector at the integrative level of processing. This would occur because bursts are typically parts of stops and, consequently, burst detectors are consulted in most phonetic environments in making phonetic decisions about stops. Since this tonic output has been changed by adaptation, it biases the output of the integrative level.

\section{EXPERIMENT 1}

Experiment 1 was an attempt to distinguish between the prototype model and the other two models, and thereby to test Diehl's second assumption. It did this by examining the transfer of adaptation from burstcued stops to transition-cued nasals.

Adaptation is said to transfer from one test continuum to another if adaptation with stimuli from one continuum affects the perception of the other continuum. Transfer of adaptation between phonetic continua based on similar acoustic cues and distinctive 
features has been demonstrated many times. For example, by varying formant transitions, it is possible to construct a phonetic continuum between [ma] and [na] whose members differ acoustically in exactly the same way as do members of a [ba-da] continuum. [m] and [n] differ in the place of articulation feature in the same way as [b] and [d]; [m] is labial, [n]. is alveolar. So it is not surprising that adaptation transfers from stops to nasals. For example, adaptation with [da] moves the [ma]-[na] boundary (Miller \& Eimas, 1977).

The three models described above explain the transfer of adaptation in different ways, and therefore, predict transfer in different circumstances. The phonetic model predicts there will be transfer whenever the adapter excites either the auditory or phonetic detectors used in the perception of the test stimuli. The auditory model predicts transfer when the test and adapting stimuli are analyzed by the same auditory detectors. The prototype model predicts transfer only when the adapter contains acoustic cues which are part of the prototypes of the test stimuli. Thus, all three models predict that adaptation with transitioncued stops will affect the perception of nasals, because the same acoustic cues and phonetic feature (the place of articulation feature) distinguish transitioncued [m] from [n] and [b] and [d].

The models differ, however, in their predictions of the effect of adaptation with a burst-cued stop on the perception of nasals. The auditory model assumes that bursts and formant transitions are analyzed by the same auditory mechanism; hence, adaptation with burst-cued stops affects the operation of the auditory analyzers used to detect the formant transitions of nasals. The phonetic model also predicts that adaptation with burst-cued stops will affect the perception of nasals, because adaptation with burstcued stops fatigues a phonetic place of articulation detector which is used to distinguish between nasals. However, the prototype model predicts that adaptation with burst-cued stops will not affect the perception of nasals. According to this model, adaptation with burst-cued stops affects burst detectors and not transition detectors. Because a burst can never occur in a nasal in naturally produced speech (the opening of the velum which causes nasalization prevents the pressure buildup behind the point of occlusion necessary to produce a burst of noise), bursts are not part of the acoustic description of nasals or the nasal prototype. Therefore, the output of burst detectors is not integrated with the output of transition detectors in deciding the place of articulation of nasals. Thus, the state of the burst detector is irrelevant to the decision as to which nasal was presented. Figure 1 is a diagram of the effect of adaptation with a burst-cued stop on the perception of both stops and nasals for the three models.

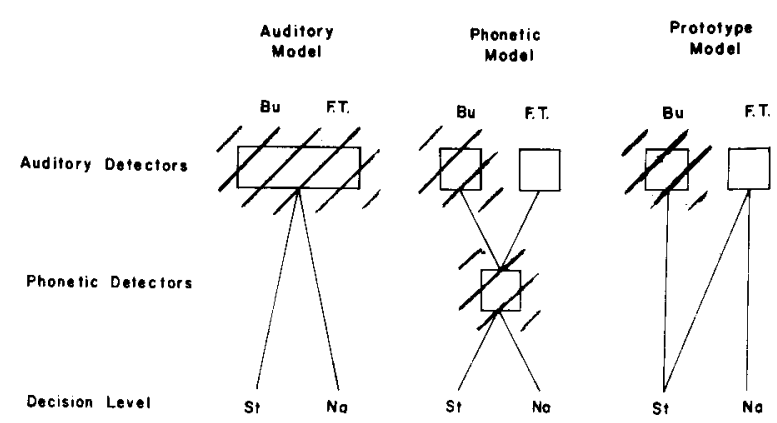

Figure 1. Information flow diagram of the three models. "Bu" and "FT" indicate sources of auditory information about bursts and formant transitions. "St" and "Na" indicate decision units for stops and nasals. Shading indicates components fatigued by adaptation with a burst-cued stop. In each model, adaptation affects the perceptions of stops, because the "stop" decision unit receives input from components affected by adaptation. The perception of nasals is unaffected by adaptation with burst-cued stops, according to the prototype model, because in just that model, the nasal decision unit receives no input from components affected by adaptation with burst-cued stops.

One way to test Diehl's second assumption is to determine whether or not adaptation with burst-cued stops affects the perception of nasals. This is the goal of Experiment 1.

It is necessary to demonstrate that any adaptation effects produced in such an experiment by the burstcued stimuli depend on the burst, and not on other aspects of its acoustic environment. Bailey (Note 3) and Wolf (Note 4) have pointed out that adaptation with steady-state vowels (which have neither bursts nor formant transitions) can affect the perception of a place of articulation test continuum. One part of the perceptual system susceptible to adaptation seems to treat the beginning of a steady-state vowel as a formant transition pattern in which the formants happen to be flat. One way to demonstrate that adaptation effects of burst-cued stops are caused by the bursts would be to show that stimuli with different bursts but the same vowel produce different adaptation effects.

Experiment 1 tested the prototype model (and, nence, Diehl's second assumption that the transfer of adaptation between different cues depends on phonetic adaptation effects) by measuring the adaptation effect of burst-cued [p] and [t] on a nasal test series. In order to provide appropriate controls and to replicate previous results, the adaptation effect of both burst- and transition-cued [p] and [t] on both stop and nasal test continua were also tested. According to all three models, adaptation with transitioncued [p] and [t] should affect the perception of both stops and nasals, and adaptation with burst-cued [p] and [t] should affect the perception of stops. The models differ in that the acoustic and phonetic models predict that adaptation with burst-cued [p] and [t] 
will affect the nasals differentially, while the prototype model predicts that there will be no differential boundary shifts produced.

A secondary goal of Experiment 1 was to investigate individual differences in subjects' phonetic categorizations of the adapting stimuli, and to determine if those differences are correlated with the direction of boundary shifts induced. Diehl (1975) reported that those subjects who labeled the burstcued stimuli as "labial" showed boundary shifts toward " $\mathrm{B}$," while those subjects who labeled them as "alveolar" showed opposite-direction boundary shifts. Sawusch and Pisoni (1976) found similar individual differences with transition-cued boundary stimuli. However, Blumstein et al. (1977) failed to replicate Diehl's findings.

\section{Method}

Stimuli. It is difficult to synthesize convincing burst-cued stops. For example, although most subjects in Diehl's (1975) study identified the burst-cued [te] correctly, many also labeled the burstcued [pe] as " $T$ " or "K." Similarly, Stevens and Blumstein (1976) found that only about one-third of their subjects identified reliably stimuli which differed only in bursts. Before high, front vowels, however, bursts are more perceptually salient than in other vowel environments (Dorman et al., 1977; Fischer-Jorgensen, Note 5). Pilot work suggested that it is possible to synthesize convincing burst-cued $[\mathrm{p}]$ and $[\mathrm{t}]$ preceding the vowel [i], so all the stimuli in this experiment were synthesized with an [i] syllable nucleus. Two test series, ranging from [bi] to [di] and from [mi] to [ni], and four adapting stimuli, burst- and transition-cued [pi] and [ti] were synthesized. All stimuli contained the same $200-\mathrm{msec}$ steady-state vowel [i]. A parametric description of the stimuli is given in the Appendix.

The [bi-di] test continuum had seven members, which differed acoustically only in their second, third, and fourth formant tran- sitions. The extreme members of the continuum had formant transitions which pilot work had indicated were appropriate for [bi] and [di]. The difference between the transitions of these stimuli was divided into equal steps, and the five other stimuli had these intermediate formant transition trajectories. The second, third, and fourth formant transitions were $60 \mathrm{msec}$ long and progressed linearly from their values at stimulus onset to their steady-state values. Pilot work had indicated that, after adaptation with voiceless stops, some subjects would often identify transitioncued [bi-di] stimuli without bursts as " $M$ " or " $N$," perhaps because of the (relatively) high first-formant starting frequency. Thus, a burst (acoustically intermediate between those appropriate for [bi] and [di]) was synthesized by adding noise filtered by a formant centered at $3,400 \mathrm{~Hz}$ to wide-band noise. An $8.9-\mathrm{msec}$ segment of this noise was spliced digitally onto the beginning of each formant transition pattern to make the [bi-di] test stimuli.

The [mi-ni] test continuum was constructed by replacing the bursts of each member of the [bi-di] test series with a nasal murmur. The murmur was synthesized by displacing the nasal pole-zero pair of the vocalic branch of the synthesizer. Except for the amplitude of voicing (which had a gradual onset), all parameters controlling the synthesizer were constant throughout the nasal murmur. This murmur was digitally spliced onto the [bi-di] test stimuli, at an upward-going zero crossing before a pitch period. Figure 2 shows schematic spectrograms of the extreme members of the two test series.

The transition-cued [pi] and [ti] adapters had exactly the same initial formant frequencies as the extreme [bi] and [di] test stimuli. In order to make the stimuli voiceless, the vocalic branch of the synthesizer was excited with aspiration, rather than voicing, for the first $40 \mathrm{msec}$ after stimulus onset and, for the same period, the bandwidth of the first formant was increased (in order to simulate "F1 cutback"). The transition-cued [pi] and [ti] had no bursts.

The burst-cued [pi] and [ti] differed only in their bursts. They both had the same formant transition pattern (one which pilot work had indicated would be identified equally dften as " $P$ " or " $T$ " when presented without a burst); the formants were excited with aspiration noise for $\mathbf{4 0} \mathrm{msec}$ before voicing onset. Following rules for speech synthesis outlined by Klatt (Note 6), the burst
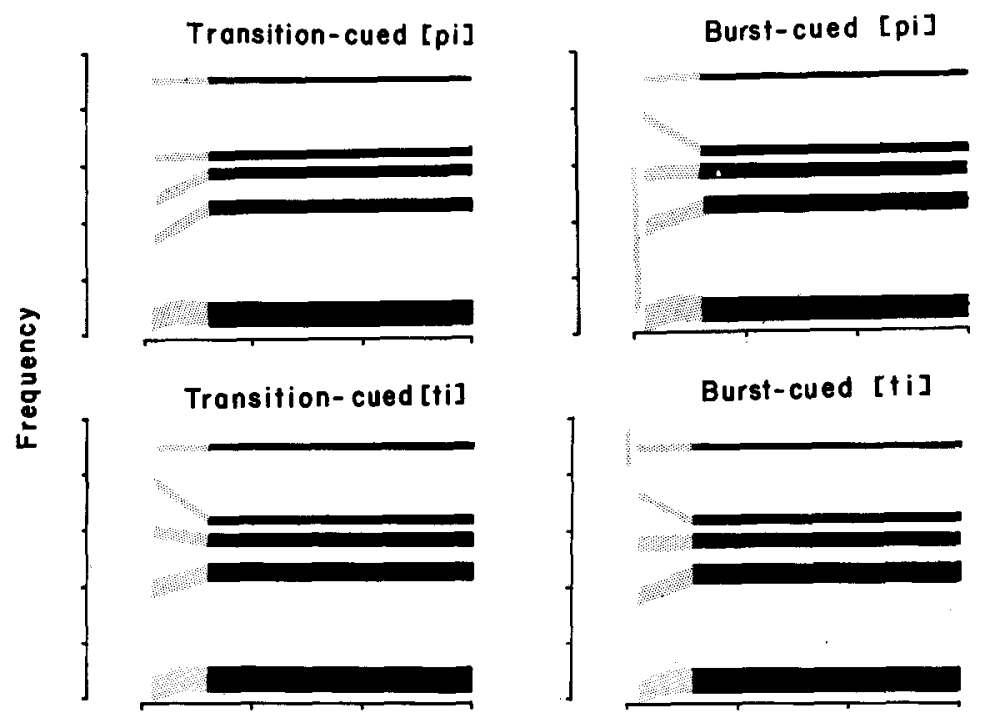

Time

Figure 2. Schematic spectrograms of the endpoints of the test continua. The row of " $O$ "'s in the nasal stimuli indicates the frequency of a zero in the vocal tract transfer function. 


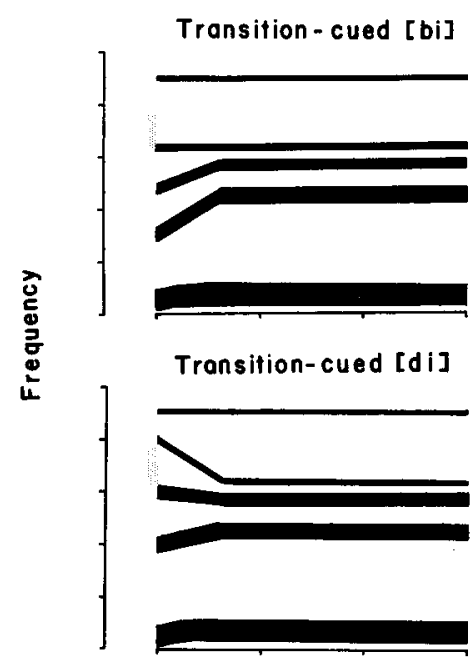

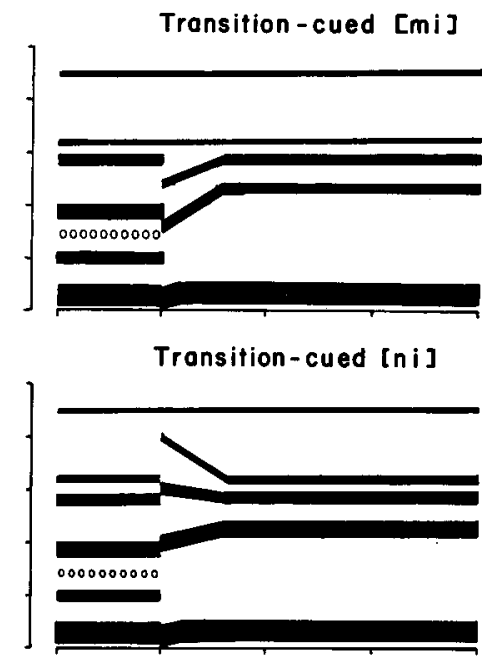

Time

Figure 3. Schematic spectrograms of the adapting stimuli. Shading indicates bursts and aspirated (rather than voiced) formants.

for the [pi] consisted of wide-band noise, while the noise for the [ti] burst was filtered by the fifth formant. Both bursts were $5 \mathrm{msec}$ long and ended $5 \mathrm{msec}$ before the onset of aspiration. Schematic spectrograms of the adapting stimuli are shown in Figure 3.

All stimuli were synthesized using a digitally simulated seriesresonance terminal-analog speech synthesizer (Klatt, 1972, Note 6). A Fortran IV version of this synthesizer was interfaced to the Psychology Department's PDP-11 computer, which synthesized all the stimuli and controlled their presentation during experimental sessions. After synthesis, all stimuli were stored digitally on the computer's disk. During the experimental sessions, the stimuli were played out using a pulse-coded modulation system, consisting of the computer's 12-bit digital-to-analog converter, a sample-and-hold circuit, and a 14-pole, elliptic low-pass filter. All stimuli were presented at $75 \mathrm{~dB}$ SPL (A scale), as measured at the headphones with a GR 1565c sound-pressure-level meter. The computer also monitored and stored subjects' responses, which were made by pressing buttons on "response boxes"- small calculators which had been modified for use with the computer.

Subjects. The subjects were 17 native speakers of American English, from the Psychology Department's subject pool. They were all M.I.T. undergraduates; 15 were male, 2 were female. They were paid for their participation in the experiment.

Procedure. Each subject participated in four sessions on different days. Each session measured the adaptation effect of one of the four adapters on both the [mi-ni] and [bi-di] test series. The subjects participated in the different sessions in different orders, and were run in groups of one to five subjects per session. An attempt was made to counterbalance the order of sessions, but perfect counterbalancing was not maintained in the face of scheduling difficulties.

In each session, there were practice, identification, and adaptation blocks. Each session began with a practice block, in which the subjects identified five repetitions of the 14 test stimuli (seven stops and seven nasals), presented in a pseudorandom order, at a rate of one every $3 \mathrm{sec}$. The subjects responded by pushing buttons labeled $\mathrm{B}, \mathrm{D}, \mathrm{M}$, or $\mathrm{N}$ on the response boxes. However, these responses were not recorded during the practice block.

The second block of each session was an "identification test," in which subjects identified 10 repetitions of each of the 14 test stimuli. The subjects again indicated their identifications of the stimuli by pushing buttons on the response boxes. These responses were recorded for later analysis.

The final block of each session measured the subjects' identifications of the test stimuli after adaptation with one of the adapting stimuli. Again, the subjects identified 10 repetitions of the [bi-di] and [mi-ni] test stimuli (presented in a pseudorandom order), but this time, before each test stimulus, the adapting stimulus was repeated 16 times (at a rate of one every $367 \mathrm{msec}$ ). The test stimulus was played $700 \mathrm{msec}$ after the last repetition of the adapting sound. Then there was a $2.7-\mathrm{sec}$ response period, after which the adapting sound started repeating again. Halfway through the adaptation block, the subjects were given a short break and asked to describe in writing the adapting sound-both to indicate whether it sounded more like $p, t, k, b, d, g, f, v$, $\mathrm{s}$, or $\mathrm{z}$ at the beginning of the adaptation session and to describe some of the verbal transformations in which it participated (Warren, 1968).

\section{Results and Discussion}

The subjects' responses were collated into histograms. These histograms were first examined to determine whether the subjects had correctly identified the manner of articulation of the test stimuli. (Since both stops and nasals were presented in each block, the subjects could have incorrectly identified the stops as $\mathbf{M}$ or $\mathbf{N}$, or the nasals as $\mathbf{B}$ or $\mathbf{D}$ ). Fourteen of the 17 subjects made very few of these errors; each of these 14 subjects made fewer than seven errors in the whole experiment. This represents an error rate of less than $.7 \%$, and these errors seem to be randomly distributed. The other 3 subjects, who had error rates as high as $10 \%$, never made enough errors to obscure the position of phoneme boundaries. Subsequent analyses were performed ignoring any response with incorrect manner of articulation.

Phoneme boundaries were calculated, using the 
method of probit analysis (Goulden, 1952) for both test continua, both before and after adaptation for each subject in each session. The phoneme boundary is the point on the stimulus continuum which divides the stimuli into separate phonetic categories; probit analysis computes a maximum likelihood estimate of the position of that point. Boundary shifts, i.e., the difference between pre- and postadaptation boundaries within each session, were also calculated. The significances of all boundary shifts were assessed with one-tailed correlated t tests with 16 degrees of freedom. Table 1 shows the mean phoneme boundaries and boundary shifts for each condition. In Table 1, boundaries and boundary shifts are given as positions along the test continua. Stimulus 1 was a [bi] or [mi], Stimulus 7, [di] or [ni]. Positive boundary shifts indicate boundary movement toward the [di] or [ni] end of the continuum, and thus represent a decrease in the number of " $D$ "' or " $N$ " responses.

As expected, the transition-cued stimuli produced substantial adaptation effects on both test continua. The transition-cued [pi] affected both the [bi-di] and [mi-ni] continua significantly $(\mathrm{t}=5.67, \mathrm{t}=5.75$, $\mathrm{p}<.001$, in each case). The transition-cued [ti] also affected both test continua significantly $(\mathrm{t}=12.32$ for [bi-di], $t=4.81$ for [mi-ni], $p<.001$, in each case). Each of these adapters affected the stop continuum more than the nasal continuum $(t=2.85$, $p<.01$ for [pi], $t=4.14, p<.001$ for [ti]). Thus, these results replicate the findings of Miller and Eimas (1977), that adaptation with transition-cued stops transfers to nasals, although not completely. In fact, about half of the boundary shift transferred in this experiment.

Adaptation with the burst-cued [ti] affected the perception of both the [bi-di] and [mi-ni] test series significantly in the predicted direction $(t=2.72$, $\mathrm{p}<.01$, for the stops, $\mathrm{t}=4.09, \mathrm{p}<.001$, for the nasals). The size of the boundary shifts on the two test continua were not significantly different. This result replicates the findings of Diehl (1975), Ainsworth (Note 1), and Ganong (Note 2) in demonstrating the effectiveness of adaptation with a "burstcued $t$ " on a transition-cued stop test series and extends this finding to nasals. This result-that adaptation with the burst-cued [ti] would affect the perception of the nasals-is predicted by the auditory and phonetic, but not prototype models.

The adaptation effects of the burst-cued [pi] were weaker and less consistent. Adaptation with it shifted the [bi-di] boundary a small (.18 stimulus units), but significant $(t=2.95, p<.01)$, amount in the predicted direction. The weak adaptation effect of the burst-cued [pi] is in accord with previous attempts to manipulate bursts as cues to place of articulation. In Diehl's (1975) study, the "burst-cued p" produced small and inconsistent boundary shifts. For labial
Table 1

Results for Experiment 1: Means and Standard Deviations of Preadaptation Boundaries and Boundary Shifts

\begin{tabular}{|c|c|c|c|c|c|}
\hline & & \multicolumn{2}{|c|}{ Boundary } & \multicolumn{2}{|c|}{ Shift } \\
\hline & & Mean & SD & Mean & SD \\
\hline Transition-Cued [pi] & $\begin{array}{l}\text { [bi-di] } \\
\text { [mi-ni] }\end{array}$ & $\begin{array}{l}4.23 \\
4.30\end{array}$ & $\begin{array}{l}.43 \\
.38\end{array}$ & $\begin{array}{l}-.86 * * \\
-.52 * *\end{array}$ & $\begin{array}{l}.62 \\
.38\end{array}$ \\
\hline Transition-Cued [ti] & $\begin{array}{l}{[\text { bi-di }]} \\
{[\text { mi-ni] }}\end{array}$ & $\begin{array}{l}4.34 \\
4.27\end{array}$ & $\begin{array}{l}.40 \\
.33\end{array}$ & $\begin{array}{l}.81^{* *} \\
.41^{* *}\end{array}$ & $\begin{array}{l}.27 \\
.35\end{array}$ \\
\hline Burst-Cued [pi] & $\begin{array}{l}{[\mathrm{bi}-\mathrm{di}]} \\
{[\mathrm{mi}-\mathrm{ni}]}\end{array}$ & $\begin{array}{l}4.32 \\
4.24\end{array}$ & $\begin{array}{l}.26 \\
.25\end{array}$ & $\begin{array}{c}-.18^{*} \\
.11\end{array}$ & $\begin{array}{l}.25 \\
.28\end{array}$ \\
\hline Burst-Cued [ti] & $\begin{array}{l}\text { [bi-di] } \\
\text { [mi-ni] }\end{array}$ & $\begin{array}{l}4.40 \\
4.25\end{array}$ & $\begin{array}{l}.47 \\
.30\end{array}$ & $\begin{array}{l}.36^{*} \\
.28 * *\end{array}$ & $\begin{array}{l}.55 \\
.29\end{array}$ \\
\hline
\end{tabular}

${ }^{*} p<.01$

${ }^{* *} p<.001$

stops, bursts may be perceptually much less salient cues for place of articulation than formant transitions (Dorman et al., 1977; Fischer-Jorgensen, Note 5).

The effect of adaptation with the burst-cued [pi] on the nasals is consistent with this pattern. Adaptation with the burst-cued [pi] moved the [mi-ni] boundary .11 stimulus units (in the wrong direction), an amount which is not significantly different from zero. This failure to significantly affect the nasal continuum may be due to splitting a small effect into insignificance. Each adapter of the present experiment affects the stop continuum more than the nasal continuum, presumably because [pi] and [ti] are stops. Thus, it is not surprising that the small adaptation effect of burst-cued [pi] on the stops did not transfer significantly to the nasal continuum.

Adaptation with the burst-cued [pi] and [ti] shifted the [mi-ni] boundary different amounts. Adaptation with [ti] shifted the boundary .28 stimulus units toward "N," while the burst-cued [pi] shifted the boundary .11 stimulus units in the same direction. The difference between these two boundary shifts (.17 stimulus units) is significantly different from zero $(t=1.97, p<.05)$. Thus, different bursts produce different adaptation effects on the nasal continuum, contradicting the prediction of the prototype model. This interpretation is strengthened by examination of the pre- and postadaptation phoneme boundaries. While the preadaptation boundaries are in the same position (the difference between them was only .01 stimulus units, $t=.14, \mathrm{p}>.10$ ), the difference in the postadaptation boundaries $(.18$ stimulus units) was significantly different from zero $(\mathrm{t}=3.15, \mathrm{p}<.01)$.

Subjects' phonetic categorizations of the adapting stimuli were next examined for correlations between individual differences in the identification of the adapting stimuli and the size of boundary shifts induced. Individual differences in subjects' descriptions of the adapting sound did not correlate with 
the direction of boundary shifts produced. Almost all subjects labeled the transition-cued [pi]s and [ti]s appropriately, so there were no individual differences in the identification of those adapting stimuli to correlate with the direction of boundary shifts. Subjects' labelings of the burst-cued stimuli were much less uniform, but still did not correlate with the direction of the boundary shifts induced by adaptation. Eleven of the subjects identified the burst-cued [pi] as "P," while four identified it as "T." The mean boundary shift among those subjects who identified it as " $T$ " was .29 stimulus units, in the same direction and not significantly different from the mean boundary shift of the other subjects $(.11$ stimulus units). Similarly, six subjects identified the burst-cued [ti] as " $P$," but they also showed approximately the same boundary shift (.37 vs. .35 stimulus units) as the other subjects. Thus, individual differences in subjects' identifications of the adapting sound had no detectable effect on the adaptation effects produced. This result conflicts with the findings of Diehl (1975) and Sawusch and Pisoni (1976) and agrees with those of Blumstein et al. (1977).

This result seems, prima facie, to support the auditory model. It seems reasonable to assume that if adaptation operates at a phonetic level, individual differences in the phonetic identification of adapting stimuli should lead to individual differences in adaptation effects. However, this assumption is not necessarily correct; individual differences in phonetic identifications [such as those observed by Sawusch and Pisoni (1976)] could just as well be caused by differences in auditory processing, and these differences could also cause differences in adaptation effects. Thus, until the causes of individual differences in phonetic identification are understood, it is premature to draw conclusions about the site of adaptation from these data.

Experiment 1's demonstration that adaptation with burst-cued [pi] and [ti] affects the perception of the [mi-ni] test series differently falsifies a prediction of the prototype model, and thus provides no evidence against Diehl's assumption that the transfer of adaptation between different acoustic cues is mediated by phonetic adaptation effects. Thus, this result is consistent with a phonetic model for Diehl's results. However, it is also consistent with the auditory model described above.

\section{EXPERIMENT 2}

The purpose of Experiment 2 was to test these two models and thereby test Diehl's assumption that information about bursts and formant transitions are not integrated at an auditory level of processing. Sawusch $(1976,1977)$ has developed an experimental technique which seems able to determine whether the integration of information about bursts and formant transitions occurs at an early or late stage of perceptual processing.

Sawusch hypothesized that the earliest stage of auditory processing of speech consists of frequencyspecific auditory detectors which are sensitive to acoustic energy within one critical band, and that a later stage is responsible for integrating information from different critical bands. To test this, he made a test series (ranging from [bae] to [dae]) and measured the adaptation effect of the endpoint stimuli. Then he measured the adaptation effect of two new stimuli, which he synthesized by shifting up in frequency each of the first three formants of the endpoint stimuli one-third of an octave (approximately one critical band). These new stimuli (which he called the "high" stimuli) produced about half as large an adaptation effect on the test continuum as did the "low" adapters (which were the endpoints of the test continuum). From this, Sawusch concluded that one-half of the adaptation effect produced by the "low" stimuli was due to the fatigue of a frequency(or critical band-) specific component, and one-half was due to a frequency-independent component which integrated the information from different frequency-specific detectors. Adaptation with the "high" adapters influenced the perception of the "low" stimuli only at this second stage of processing, and therefore, produced only half the effect of the "low" adapters.

In order to determine the anatomical locus of these two stages of processing, Sawusch next measured the interaural transfer of adaptation effects produced with the high and low stimuli. The adaptation effect of the "high" stimuli (on the low test continuum) transferred interaurally $100 \%$-when he presented the adapting stimuli to one ear, the boundary shifts induced were the same, whether he tested in the adapted or unadapted ear. On the other hand, the adaptation effect of the "low" stimuli (the endpoints of the test continuum) transferred interaurally only $50 \%$; the boundary shift on the unadapted ear was only half that produced on the adapted ear, and was equal to the boundary shift induced by the "high" adapters. Sawusch concluded that the frequencyspecific processing stage is peripheral (i.e., one such processor is associated with each ear), and that the frequency-independent processing stage is central and sensitive to information from both ears.

It is easy to see how this technique can be used to determine whether the adaptation effects of burstcued stops occur at early or late stages of perceptual processing. If bursts and transitions are detected by the same low-level peripheral auditory mechanism, then the adaptation effect of the burst-cued stimuli should transfer interaurally to the same degree as the adaptation effect of the transition-cued stimuli. On 
the other hand, if adaptation with burst-cued stimuli affects the perception of transition-cued stimuli at the later, frequency-independent level of processing, the adaptation effect of burst-cued stops must transfer interaurally completely. Since phonetic categorization is not affected by raising formants one critical band, by definition, any phonetic processes must be frequency-independent. Hence, if adaptation with burst-cued stops affects the perception of transition-cued stops at a phonetic level of processing, complete interaural transfer of their adaptation effects would be expected.

\section{Method \\ Subjects. Twelve men and one woman from the Psychology Department's subject pool participated in the experiment. Four of them had participated in Experiment 1. \\ Stimuli. The same test stimuli were used as in Experiment 1. Because the burst-cued (pi] was not an effective adapter in Experiment 1, only the adaptation effects of the burst- and transition-cued [ti] were assessed in Experiment 2. \\ Stimuli were again presented using the pulse-coded modulation system described above, but the output of that system was directed to a set of computer-controlled relays. The relays were set so that each channel either received the signal the computer was playing out or was grounded, so as to produce silence. These two channels were amplified and presented to subjects through headphones. All stimuli were presented at $75 \mathrm{~dB}$ SPL (A scale) as measured at the headphones. \\ Procedure. Each subject participated in four sessions. Each session determined the adaptation effect on both ears of one of the two adapters on either the nasal or stop test continuum. As before, each session consisted of practice, identification, and adaptation blocks. The format of each block was similar to the format of the analogous block of Experiment 1. Within each block, subjects were presented with test stimuli on either ear. In order to counterbalance for any differences between the two channels, subjects reversed their headphones halfway through each block. Seven of the subjects listened to the adapting stimulus with the right ear, six with the left.}

\section{Results and Discussion}

The data from Experiment 2 were analyzed in exactly the same manner as those from Experiment 1. Phoneme boundaries and boundary shifts were calculated using probit analysis, and the significance of boundary shifts were assessed with one-tailed $t$ tests with 12 degrees of freedom. Results of these calculations are shown in Table 2.
Adaptation with the transition-cued [ti] shifted the [bi-di] boundary significantly on both the adapted and unadapted ears (shift $=.94$ and .30 stimulus units, $\mathrm{t}=7.44$ and $2.36, \mathrm{p}<.001$ and $\mathrm{p}<.05$, respectively). The shift produced on the unadapted ear was about one-third the shift produced on the adapted ear and was significantly less $(t=3.00$, $p<.01)$ than the shift on the adapted ear. This result replicates Ades' and Sawusch's findings that adaptation with transition-cued stimuli transfers interaurally less than $100 \%$.

The pattern of results produced by adaptation with the transition-cued [ti] on the nasals is similar. Phoneme boundaries on both the adapted and unadapted ears were shifted significantly in the predicted direction $(\mathrm{t}=5.34$ and 2.81, $\mathrm{p}<.001$ and $\mathrm{p}<.01$ ); again the boundary shift on the unadapted ear was about one-third of that on the adapted ear (.14 vs. .57 stimulus units), and, again, the boundary shifts on the two ears were significantly different $(t=4.58, p<.001)$. Thus, the adaptation effect of transition-cued [ti] seems to transfer interaurally to the same degree for nasals and stops, suggesting that adaptation affects the nasals at the same level of processing as it affects the stops.

The adaptation effects of the burst-cued [ti] on the [bi-di] series also transferred interaurally about onethird. Again, the boundary shifts on both ears were significant $(t=8.93$ and $2.54, \mathrm{p}<.001, \mathrm{p}<.05)$, and, again, the boundary shifts on the unadapted ear were significantly less $(t=4.16, p<.01)$ than the boundary shifts on the adapted ear. This finding suggests that the adaptation effects produced by the burst-cued [ti] are produced at the same low level of processing as the adaptation effects produced by the transition-cued [ti].

Finally, adaptation with the burst-cued [ti] did not affect the perception of the nasal test series significantly on either ear. The boundary shift induced on the adapted ear was in the expected direction, but was not quite significant $(t=1.49, \mathrm{p}<.1)$. On the unadapted ear, the mean boundary shift was zero. The pattern of boundary shifts in this condition is similar to that in other conditions, but the boundary

Table 2

Results for Experiment 2: Means and Standard Deviations of Preadaption Boundaries and Boundary Shif ts Following Adaptation with Either Burst- or Transition-Cued [ti]

\begin{tabular}{|c|c|c|c|c|c|c|c|c|c|c|}
\hline \multirow[b]{3}{*}{ Adaptation } & & & \multicolumn{4}{|c|}{ Adapted Ear } & \multicolumn{4}{|c|}{ Unadapted Ear } \\
\hline & & & \multicolumn{2}{|c|}{ Boundary } & \multicolumn{2}{|c|}{ Shift } & \multicolumn{2}{|c|}{ Boundary } & \multicolumn{2}{|c|}{ Shift } \\
\hline & & & Mean & SD & Mean & SD & Mean & SD & Mean & SD \\
\hline Transition-Cued [ti] & $\begin{array}{l}\text { Test } \\
\text { Series }\end{array}$ & $\begin{array}{l}\text { [bi-di] } \\
\text { [mi-ni] }\end{array}$ & $\begin{array}{l}4.13 \\
4.07\end{array}$ & $\begin{array}{l}.52 \\
.46\end{array}$ & $\begin{array}{l}.94 \dagger \\
.57 \dagger\end{array}$ & $\begin{array}{l}.46 \\
.39\end{array}$ & $\begin{array}{l}4.26 \\
4.22\end{array}$ & $\begin{array}{l}.51 \\
.30\end{array}$ & $\begin{array}{l}.30^{*} \\
.14^{* *}\end{array}$ & $\begin{array}{l}.45 \\
.18\end{array}$ \\
\hline Burst-Cued [ti] & $\begin{array}{l}\text { Test } \\
\text { Series }\end{array}$ & $\begin{array}{l}\text { [bi-di] } \\
\text { [mi-ni] }\end{array}$ & $\begin{array}{l}4.19 \\
4.24\end{array}$ & $\begin{array}{l}.39 \\
.33\end{array}$ & $\begin{array}{l}.53 \dagger \\
.11\end{array}$ & $\begin{array}{l}.21 \\
.26\end{array}$ & $\begin{array}{l}4.09 \\
4.35\end{array}$ & $\begin{array}{l}.51 \\
.27\end{array}$ & $\begin{array}{c}.15^{*} \\
0\end{array}$ & $\begin{array}{l}.22 \\
.23\end{array}$ \\
\hline
\end{tabular}

${ }^{*} p<.05 \quad{ }^{* *} p<.01 \quad t_{p}<.0001$ 
shifts are not large enough to reach significance.

This failure to replicate the results of the crucial condition of Experiment 1 (which tested the adaptation effect of burst-cued stimuli on the nasals) is unfortunate, but does not demonstrate that that result was a Type I error. The result obtained in Experiment 2 was smaller than, but in the same direction as, the result of Experiment 1. The smaller size of this effect may have been due to differences in procedure; in Experiment 1, all stimuli were presented to both ears simultaneously, while in Experiment 2 they were presented to one ear at a time. And the failure of that latter effect to reach significance may be a function of the smaller number of subjects used in the second experiment. In order to determine if adaptation with the burst-cued [ti] produced a significant adaptation effect on the nasals overall, the data from the two experiments were pooled. Since four subjects participated in both experiments, the analysis was carried out twiceonce including, once excluding the four "repeated" subjects. In both computations, the observed boundary shift is highly significant [with the repeated subjects included, the mean boundary shift was .14 stimulus units, $\mathrm{t}(42)=3.30, \mathrm{p}<.005$; with those subjects excluded, the mean boundary shift was .18 stimulus units, $\mathrm{t}(30)=3.74, \mathrm{p}<.001]$. Thus, the conclusion of Experiment 1-that adaptation with burst-cued [ti] affects the perception of the [mi-ni] test series-seems warranted.

These results demonstrate that adaptation with burst-cued stimuli transfers interaurally to the same degree as does adaptation with transition-cued stimuli. This is in accord with the predictions of the auditory model and disagrees with the predictions of the phonetic model, which predicted that adaptation effects of burst-cued stimuli would transfer interaurally more readily than did the adaptation effects of the transition-cued stimuli.

One potentially interesting difference between the results of this experiment and previous experiments on the interaural transfer of adaptation is that the size of the interaural transfer seems to be smaller in this experiment. While Ades (1974) and Sawusch (1976) both reported about $50 \%$ interaural transfer, interaural transfer in this experiment averaged about one-third. One plausible source for this difference is in the testing procedures used. Both Ades and Sawusch had subjects listen to many repetitions of the adapting sound, then identify a set of four to seven test stimuli. In the present experiment, subjects heard 16 repetitions of the adapting stimuli, followed by only one test stimulus. If there is a decay of adaptation effect during the test period, Ades' and Sawusch's procedures would have been more sensitive to it. Thus, the difference in results can be explained by assuming that the time course of decay of adaptation effects is different for central and peripheral adaptation effects. Another possibility is that the difference in interaural transfer is a function of stimuli used; Ades used a [bae-dae] continuum, Sawusch, a [ba-da] continuum, and the present study a [bi-di] continuum.

The results of Experiment 2 show clearly that the adaptation effect of both burst-and transition-cued stops transfers interaurally only about one-third. According to the interpretation of the results of Sawusch's experiments given above, this is clear evidence against the view that the adaptation effect of burst-cued stops is produced at a higher, that is, phonetic, level of processing. But this rejection of the phonetic model depends crucially on the interpretation of Sawusch's data. Without these data, there would be no reason to equate phonetic processing with complete interaural transfer, and no reason to equate small degrees of interaural transfer with an auditory level of processing.

Sawusch's interpretation of his result can be questioned in several ways. For example, just as interocular transfer is not a reliable index of centrallymediated visual effects, perhaps interaural transfer is not a reliable index of centrally-mediated auditory adaptation effects. Or, assuming there is a central component, is it really frequency-independent (see Bailey, 1976, for some contradictory evidence)? But these objections are irrelevant to the argument presented here. The interpretation of Experiment 2 depends solely on the assumption that any phonetic adaptation effects must show complete interaural transfer. And that assumption is directly supported by Sawusch's data. In his study, adaptation with the "high" stimulus affected the unadapted and adapted ears to the same degree. If there was a phonetic component of adaptation which did not show complete interaural transfer, it should have shown itself in this condition, because the "high" stimulus was from the same phonetic category as the "low" stimulus. Thus, while alternative explanations for Sawusch's data are possible, none of these explanations can maintain that phonetic adaptation effects do not show complete interaural transfer. And this is just the aspect of Sawusch's interpretations which is needed here. So it is safe to conclude from the results of Experiment 2 that information about bursts and transitions is not integrated at a phonetic level of processing. Thus, Diehl's second assumption and the phonetic model are incorrect.

\section{GENERAL DISCUSSION}

The results of Experiment 2 can only be explained by assuming that bursts and formant transitions are 
integrated at an auditory level of processing. Thus, Assumption 1 is incorrect, and the auditory model would seem to be the best acount of Diehl's data. However, two different mechanisms for the integration of information about bursts and formant transitions at an auditory stage of processing have been proposed. One is the "global acoustic property detector model" of Blumstein, Stevens, and Nigro (1977); the other is the frequency-specific model of Ades (1976).

Ades (1976) proposed that adaptation occurs at a level of processing that is sensitive to changes in frequency regardless of whether the changes are brought about by bursts or formant transitions. He pointed out that Diehl's burst-cued [te], which produced significant adaptation effects, contained a frequency change like that caused by the third formant of the [de] test stimuli. On the other hand, the burst-cued [pe] had a pattern of frequency change unlike that of any of the test stimuli-and produced no significant adaptation effect. However, this explanation cannot be correct, as Sawusch (1976) has pointed out. Ganong (Note 2) demonstrated adaptation effects (on a transition-cued test series) of a burst-cued [tae] which had burst center frequency at $4,500 \mathrm{~Hz}$. This is much higher than the $3,000-\mathrm{Hz}$ frequency of the third-formant transition of the most extreme test stimulus. Thus, the failure of Diehl's burst-cued [pe] adapter must not have been due simply to the difference in frequency between the burst and nearby formants.

In fact, a recent experiment by Diehl and Groves (Note 7) shows that adaptation cannot be operating at a level which simply registers frequency changes. They synthesized a two-formant [da-ga] test series, in which the second-formant starting frequency ranged from $1,560 \mathrm{~Hz}$ (for [da]) to $2,160 \mathrm{~Hz}$ (for [ga]). The second-formant steady-state frequency was $1,347 \mathrm{~Hz}$ for all stimuli. They also synthesized two burst-cued stimuli with burst center-frequencies as 1,490 and $2,420 \mathrm{~Hz}$. The stimulus with the higher frequency burst was heard as [ta]. The pattern of spectral change in this stimulus is like that of [ga], yet it had a [da]-like adaptation effect. The frequencyspecific model of adaptation predicts that the lower frequency burst should have a [da]-like effect, and the higher frequency burst, a [ga]-like effect. Neither of these results was obtained, so the frequencyspecific modcl of adaptation can be rejected as an explanation for the integration of information about bursts and formant transitions.

Another attempt to attribute the adaptation effects of burst-cued stimuli to an acoustic level of processing was made by Blumstein et al. (1977). They proposed that the perception of place of articulation was mediated by global acoustic property detectors which were sensitive to both bursts and formant transitions. The global acoustic properties to which these detectors were sensitive were assumed to be contextindependent properties of place of articulation which are not frequency specific, but which are spread throughout the spectrum. Whether such properties exist is, of course, an empirical (and still open) question, but Stevens (1975) and Stevens and Blumstein (1976) have proposed several properties which might be context-independent cues for place of articulation. One such proposed property is "onset spectrum." Another is the direction of spectral change. Blumstein et al. (1977) assume that the global acoustic property detectors integrate information about bursts and transitions, because both kinds of information are available at syllable onset, and that adaptation affects this stage of processing. Thus, their model differs from Ades' in the type of information integrated at this stage of processing, but agrees with his model in assuming that the transfer of adaptation between burst- and transition-cued stimuli is mediated by the fatigue of detectors for acoustic attributes.

Blumstein, Stevens, and Nigro's (1977) global acoustic property detector model, with slight modifications, can account for the results of the present study as well as Diehl and Groves' (Note 7) results. That model is sensitive to factors like the relative amplitude of bursts and formant transitions, and may treat bursts and formant transitions rather differently. In particular, it is possible that "global acoustic detectors" used to distinguish [d] and [t] from other stop consonants are strongly excited by the falling formant transition pattern of the [da] test stimulus and by highfrequency noise bursts at stimulus onset. Thus, the global acoustic property detector model can easily account for the results of Diehl and Groves' experiment by assuming that only the [ta] burst was at a high enough frequency to excite this detector. It can also account for the results of Experiment 1 by assuming that the acoustic property detectors which integrate information about bursts and formant transitions (in order to decide whether " $B$ " or " $D$ " was presented) is also used to distinguish between nasals. Blumstein et al. do not specify the "anatomical locus" of the global acoustic property detectors. The model can be extended to account for the results of Experiment 2 by postulating that the detectors are partially lateralized, that is, that each detector receives primary input from one ear and weakened input from the other ear.

Regardless of the details of the auditory model's explanation of the adaptation effect of burst-cued stops, it is clear that the proper explanation of these effects will involve changes in auditory processes which are sensitive to both bursts and formant tran- 
sitions. This conclusion strengthens the hypothesis that adaptation affects solely an auditory stage of processing.

\section{APPENDIX}

This appendix is a more detailed description of the way in which the stimuli for this study were constructed. The stimuli were synthesized using a 5-pole series-resonance terminal-analog speech synthesizer, simulated at a $10-\mathrm{kHz}$ sampling rate (Klatt, 1972). This synthesizer is much more flexible than needed for the synthesis of the stimuli used in this study, so many parameters were left at their default values. For instance, the bandwidths of the five formants were $50,70,110,170$, and $250 \mathrm{~Hz}$. Many other of these parameters are peculiar to this synthesizer and will not be specified here. All parameters which changed values during a stimulus changed linearly, so values are specified only at times in which they change direction or rate of change. Parameter values at intermediate times can be obtained by linear interpolation. Parameters were updated at $5-\mathrm{msec}$ intervals.

The [bi-di] and [mi-ni] test series were synthesized by adjoining a burst or a nasal murmur to syllable nuclei which varied only in formant transitions. Parameters used in the synthesis of these syllable nuclei are described in Table A.

The initial values of the second, third, and fourth formants were different for the different members of the test continua. Initial formant frequencies for Stimulus 1 (the extreme [bi] or [mi]) were $1,500,2,400$, and $3,200 \mathrm{~Hz}$ (for F2, F3, and F4); for Stimulus 7, they were 2,000, 3,000 , and $4,000 \mathrm{~Hz}$. Intermediate stimuli had intermediate formant transition starting frequencies.

A burst (for the [bi-di] test series) was synthesized by summing white noise with noise filtered by a simple resonant filter which has a center frequency of $3,400 \mathrm{~Hz}$ and a bandwidth of $340 \mathrm{~Hz}$. This burst is acoustically intermediate between those appropriate to $[\mathrm{p}]$ and $[\mathrm{t}]$. The input to this filter was left at 0 for $90 \mathrm{msec}$, then turned to $48 \mathrm{~dB}$ for $8.9 \mathrm{msec}$. A nasal murmur was synthesized by passing voicing through the vocal tract branch of the synthesizer and its nasal pole-zero pair. The formant frequencies during the nasal murmur were $300,1,900,2,840,3,200$, and $4,500 \mathrm{~Hz}$. The nasal zero was set at $1,400 \mathrm{~Hz}(150-\mathrm{Hz}$ bandwidth), while the nasal pole was set at $1,000 \mathrm{~Hz}$ (also with $250-\mathrm{Hz}$ bandwidth). The fundamental frequency of the voicing source was $140 \mathrm{~Hz}$ throughout the nasal murmur.

The [mi-ni] and [bi-di] stimuli were produced by adjoining the first $98.9 \mathrm{msec}$ of each of these two waveforms to

Table A

Parameters (P) for Syllable Nuclei of Test Stimuli

\begin{tabular}{cccccccrr}
\hline & \multicolumn{7}{c}{ Time (in Milliseconds) } \\
\cline { 2 - 8 } P & 0 & 20 & 45 & 60 & 235 & 255 & 260 & 295 \\
\hline F0 & 145 & & & & & & & 110 \\
F1 & 250 & 338 & 350 & & & & & 350 \\
F2 & $*$ & & & 2250 & & & & 2250 \\
F3 & $*$ & & & 2850 & & & & 2850 \\
F4 & $*$ & & & 3200 & & & & 3200 \\
AV & 63 & & & & 63 & 51 & 0 & 0 \\
\hline
\end{tabular}

*Different for different stimuli.
Table B

Parameters (P) the Adapters Share

\begin{tabular}{ccccrrrrrrr}
\hline & \multicolumn{8}{c}{ Time (in Milliseconds) } \\
\cline { 2 - 10 } P & 0 & 10 & 30 & 50 & 55 & 60 & 235 & 255 & 260 & 295 \\
\hline F0 & 145 & & & & & & & & \\
F1 & 250 & 250 & 338 & & & 350 & & & 110 \\
F2 & $*$ & $*$ & & & & 2250 & & & & 2250 \\
F3 & $*$ & $*$ & & & & 2850 & & & & 2850 \\
F4 & $*$ & $*$ & & & & 3200 & & & & 3200 \\
AV & 0 & & & 0 & 63 & & 63 & 51 & 0 & 0 \\
AH & 0 & 53 & & 53 & 0 & & & & & 0 \\
BW1 & 100 & & & 100 & 50 & & & & & 50 \\
\hline
\end{tabular}

*Different for different stimuli.

Table C

Starting Frequencies for Formant Transitions for Adapters

\begin{tabular}{lccc}
\hline & \multicolumn{3}{c}{ Parameter } \\
\cline { 2 - 4 } \multicolumn{1}{c}{ Stimulus } & F2 & F3 & F4 \\
\hline Transition-Cued [pi] & 1500 & 2400 & 3200 \\
Transition-Cued [ti] & 2000 & 3000 & 4000 \\
Burst-Cued [pi] & 1875 & 2850 & 3800 \\
Burst-Cued [ti] & 1875 & 2850 & 3800 \\
\hline
\end{tabular}

the syllable nuclei containing the formant transitions. This particular duration $(98.9 \mathrm{msec})$ was chosen because it represents the last upward-going zero crossing before a pitch period in the nasal murmur.

The adapting stimuli shared many of their parameter values. Table B is a description of the values they had in common. These stimuli differed only in the starting values of the formant frequencies (which are given in Table C) and the bursts.

The transition-cued stimuli had no bursts. The burstcued stimuli had 5 -msec bursts, which ended 5 msec before the onset of aspiration. The burst amplitude parameter was set equal to 54 during the burst. The spectrum of the burst for [pi] was shaped by a simple low-pass digital resonator with a bandwidth of $3,000 \mathrm{~Hz}$. The [ti]'s burst was shaped by a resonant filter with a $4,500-\mathrm{Hz}$ center frequency and a 250-Hz bandwidth.

\section{REFERENCE NOTES}

1. Ainsworth, W. A. Some effects of adaptation on phoneme boundaries. Paper presented at the Eighth International Congress of Phonetic Sciences, Leeds, England, 1975.

2. Ganong, W. F. An experiment on "phonetic adaptation." Progress Report, Research Laboratory of Electronics, Massachusetts Institute of Technology, 1975, 116, 206-210.

3. Bailey, P. J. Personal communication, June 1976.

4. Wolf, C. G. Perceptual invariance for stop consonants in initial and final position. Manuscript in prepration, 1977.

5. Fischer-Jorgenson, E. Perceptual studies of Danish stop consonants. Annual Report of the Institute of Phonetics, University of Copenhagen, 1972, 75-128.

6. Klatt, D. H. Cascade/parallel terminal-analog speech synthesizer and a strategy for consonant-vowel synthesis. Manuscript in preparation, 1977.

7. Dieh1, R. L., \& Groves, K. A. Selective adaptation to speech: Is there a phonetic component? Manuscript submitted for publication, 1977. 


\section{REFERENCES}

ADEs, A. E. Bilateral component in speech perception? Journal of the Acoustical Society of America, 1974, 56, 610-616.

ADEs, A. E. Adapting the property detectors for speech perception. In R. Wales \& E. Walker (Eds.), New approaches to language mechanisms. Amsterdam: North Holland, 1976.

BaILEY, P. J. Perceptual adaptation in speech: Some properties of detectors for acoustical cues to phonetic distinctions. Unpublished doctoral dissertation, Cambridge University, 1975.

BarLow, H. B. Single units and sensation: A neuron doctrine for perceptual psychology? Perception, 1972, 1, 371-394.

Blumstein, S. E., Stevens, K. N., \& Nigro, G. N. Property detectors for bursts and transitions in speech perception. Journal of the Acoustical Society of America, 1977, 61, $1301-1313$

CoOper, W. E. Selective adaptation for acoustic cues of voicing in initial stops. Journal of Phonetics, 1974, 2, 303-314.

$D_{A}, R$. H. On interocular transfer and the central origin of visual aftereffects. American Journal of Psychology, 1958, 71, 784.790.

Delabarre, E. B. On the seat of optical after-images. American Journal of Psychology, 1888, 2, 326-328.

DiEHL, R. L. The effect of selective adaptation on the identification of speech sounds. Perception \& Psychophysics, 1975, 17, 48-52.

Dorman, M. F., Studdert-Kennedy, M., \& Raphael, L. J. Stop-consonant recognition: Release bursts and formant transitions as functionally equivalent, context-dependent cues. Perception \& Psychophysics, 1977, 22, 109-122.

Eimas, P. D., \& Corbit, J. D. Selective adaptation of linguistic feature detectors. Cognitive Psychology, 1973, 4, 99-109.

Ganong, W. F. Amplitude contingent selective adaptation to speech. Journal of the Acoustical Society of America, 1976, 59, S26. (A).

Goulden, C. H. Methods of statistical analysis (2nd ed.). New York: Wiley, 1952.

HoffmaN. H, S. Study of some cues in the perception of voiced stop consonants. Journal of the Acoustical Society of America, 1958, 30, 1035-1041.

KLATT, D. H. Acoustic theory of terminal analog speech synthesis. Proceedings 1972 Conference on Speech Communication and Processing, April 24-26, 1972. Boston, Massachusetts, IEEE Catalog No. 72 CHO 596-7 AE, 131-134.

LISKER, L. Is it VOT or a first-formant transition detector? Journal of the Acoustical Society of America, 1975, 57, 1547-1551.

Miller, J. L., \& Einias, P. D. Studies on the perception of place and manner of articulation: A comparison of the labial-alveolar and nasal-stop distinctions. Journal of the Acoustical Society of America, 1977, 61, 835-845.

Parker, F. Distinctive features and acoustic cues. Journal of the Acoustical Society of America, 1977, 62, 1051-1054.

SAWUSCH, J. R. The structure and flow of information in speech perception: Evidence from selective adaptation of stop consonants. Unpublished doctoral dissertation, Indiana University, 1976.

SAWUSCH, J. R. Peripheral and central processes in selective adaptation of place of articulation in stop consonants. Journal of the Acoustical Society of America, 1977, 62, 738-750.

SAWusch, J. R., \& Pisoni, D. B. Response organization in selective adaptation to speech sounds. Perception \& Psychophysics, 1976, 20, 413-418.

STEvens, K. N. The potential role of property detectors in the perception of consonants. In G. Fant \& M. A. Tatham (Eds.), Auditory analysis and perception of speech. London: Academic Press, 1975.

Stevens, K. N., \& Blumstein, S. E. Context-independent properties for place of articulation in stop consonants. Journal of the Acoustical Society of America, 1976, 59, S40. (A)

WARREN, R. M. Verbal transformation effect and auditory perceptual mechanisms. Psychological Bulletin, 1968, 70, 261-270.

\section{NOTES}

1. There has been some disagreement about the meaning of the phrase "phonetic level of processing." This paper, like that of Diehl (1975), uses the phrase to refer to a stage of processing which is sensitive to the particular phonetic category of a stimulus.

2. Cooper (1974) carried out a similar adaptation study of two acoustic cues (voice onset time and duration of formant transitions) for the voicing distinction in English syllable initial stops. Like Diehl, he found that adaptation with stimuli defined by one cue affected the perception of stimuli defined by the other cue. However, he noted that transition duration and voice onset time need not be different cues, in the sense of being detected by different mechanisms, and subsequent research has shown he was correct. Both VOT and transition duration seem to be detected by lowlevel detectors for something like first formant starting frequency (Lisker, 1975).

(Received for publication November 18, 1977; revision accepted May 18, 1978.) 